\title{
Do Behavioral Risk Factors for Prediabetes and Insulin Resistance Differ across the Socioeconomic Gradient? Results from a Community-Based Epidemiologic Survey
}

\author{
May H. Yang, ${ }^{1}$ Sue A. Hall, ${ }^{1}$ Rebecca S. Piccolo, ${ }^{1}$ Nancy N. Maserejian, ${ }^{1}$ \\ and John B. McKinlay ${ }^{2}$ \\ ${ }^{1}$ New England Research Institutes, Inc., Watertown, MA 02472, USA \\ ${ }^{2}$ Department of Epidemiology, New England Research Institutes, Inc., 480 Pleasant Street, Suite 100A, Watertown, \\ MA 02472, USA \\ Correspondence should be addressed to John B. McKinlay; jmckinlay@neriscience.com
}

Received 22 August 2014; Accepted 29 September 2014

Academic Editor: Ilias Migdalis

Copyright (C) 2015 May H. Yang et al. This is an open access article distributed under the Creative Commons Attribution License, which permits unrestricted use, distribution, and reproduction in any medium, provided the original work is properly cited.

\begin{abstract}
To examine whether behavioral risk factors associated with diabetes (diet, BMI, waist circumference, physical activity, and sleep duration) are also related to both prediabetes and insulin resistance (IR), we used data from Boston Area Community Health (BACH) Survey $(2010-2012, n=3155)$. Logistic and linear regression models were used to test the association of lifestyle factors with prediabetes status, insulin resistance, and prediabetes or insulin resistance. All regression models were stratified by education and income levels (to examine whether risk factors had differential effects across socioeconomic factors) and adjusted for age, gender, race/ethnicity, family history of diabetes, and smoking status. We found that large waist circumference was consistently associated with higher levels of insulin resistance (IR) and increased odds of prediabetes. While the association between large waist circumference and IR was consistent across all levels of SES $(P<0.001)$, the association between large waist circumference and prediabetes was only statistically significant in the highest socioeconomic strata with odds ratios of 1.68 (95\% CI 1.07-2.62) and 1.88 (95\% CI 1.22-2.92) for postgraduate degree and income strata, respectively. There was no association between diet, physical activity, sleep duration, and the presence of multiple risk factors and prediabetes or IR within SES strata.
\end{abstract}

\section{Introduction}

The prevalence of type 2 diabetes continues to rise and currently affects over 25 million Americans and is estimated to reach 439 million adults worldwide by 2030 [1, 2]. Along with this increase, the prevalence of prediabetes is also rising, affecting approximately one in three U.S. adults aged $\geq 20$ years (an estimated 79 million individuals) [3, 4]. Furthermore, it is estimated that, among U.S. adults with undiagnosed diabetes or impaired fasting glucose, the proportion of adults with insulin resistance increased from $24.8 \%$ to $31.1 \%$ during the time periods of 1988-1994 to 19992002 [5].

Prediabetes is indicated when blood glucose or hemoglobin Alc (Alc) levels are higher than normal, but not yet high enough to be classified as diabetes. Prediabetes raises the risk of type 2 diabetes by 3 - to 10 -fold and it is estimated that up to $70 \%$ of people with prediabetes may develop type 2 diabetes during their lifetime [3, 6, 7]. A study conducted by Geiss et al. [8] estimates $30 \%$ of the U.S. adult population had prediabetes in 2005-2006, but only 7\% were aware that they had the condition. Insulin resistance (IR) is a condition in which the body produces insulin but does not use it effectively. When people have insulin resistance, glucose builds up in the blood instead of being absorbed by the cells, leading to type 2 diabetes or prediabetes.

The American Diabetes Association (ADA) (April 4) [9] considers both prediabetes and insulin resistance precursors to type 2 diabetes. Both the ADA and the National Heart Lung and Blood Institute's National Cholesterol Education 
Program (NCEP) [10] suggest the risk factors for type 2 diabetes, prediabetes, and IR are similar and include the following (not comprehensive): obesity, physical inactivity, family history of diabetes, race/ethnicity, high blood pressure, large waist circumference, and high triglycerides. Notably, socioeconomic status is usually not included but is an important focus of this paper.

Socioeconomic status is a complex construct, broadly based on relative income, education, and occupation-all of which have been repeatedly associated with lifestyles and behavioral risk factors. In this analysis, we use income and education as indicators of SES, since occupation has been shown to be highly correlated with education $[11,12]$. Numerous studies have identified low physical activity [13-15], low diet quality $[14,16,17]$, and, more recently, poor sleep quality, as associated with diabetes [18-22]. However, it is uncertain whether these factors equally affect prediabetes and diabetes risk across the SES gradient. That is, most previous studies statistically adjust for education, income, or some other marker of SES [23-25]. But to develop well-targeted and likely effective primary and secondary interventions it is necessary to understand how modifiable risk factors influence risk of diabetes among the different SES groups. Studies have shown that while health programs and therapies exist to manage prevention of diabetes and its complications, these programs are underutilized among those in low socioeconomic groups [26-28].

While there are many risk factors contributing to diabetes, this study seeks to identify those most likely to improve the effectiveness of primary and secondary prevention initiatives. Coupled with the under-utilization of diabetes prevention programs among those with low SES [26-28], there is a potential benefit to identifying modifiable diabetes risk factors that may contribute to prediabetes among those with low SES and to examine the different effects of diabetes risk factors operating among different SES levels.

Therefore, the objectives of this analysis are

(1) within and across each level of SES, to examine the associations between known behavioral risk factors for diabetes (low diet quality, poor sleep, low physical activity, and high waist circumference) and both prediabetes and IR;

(2) to examine these modifiable factors collectively (presence of two or more risk factors) for their potential impact on the prevalence of prediabetes and IR within and across the SES gradient.

\section{Materials and Methods}

2.1. Participants. The Boston Area Community Health $(\mathrm{BACH})$ Survey is a longitudinal cohort study of residents of Boston, MA, aged 30-79 years at baseline (March 2002June 2005). Detailed methods have been described elsewhere $[29,30]$. Briefly, a stratified two-stage cluster sample was used to recruit an approximately equal number of participants by gender, race/ethnicity (Black, Hispanic, White), and age group (30-39, 40-49, 50-59, 60-79). 5,502 adults participated in baseline BACH I (1767 Black, 1876 Hispanic, 1859 White;
2301 men, 3201 women). Follow-up surveys were collected at two time points approximately 5 (BACH II 2006-2010) and 7 (BACH III 2010-2012) years later. For BACH III, completed interviews were obtained for 3155 individuals (1184 men; 1971 women). In all surveys, data were collected during a twohour interview in English or Spanish, after obtaining written informed consent. The study was approved by New England Research Institutes' Institutional Review Board. Analyses for this paper use data from the most recent interview, BACH III (2010-2012).

2.2. Sociodemographic Lifestyle Assessment Measures. Education, based on years of education completed, is composed of four categories: <high school, high school or GED, some college, or college or advanced degree. Income was collected as total annual income and categorized into three categories: $<\$ 20,000, \$ 20,000-\$ 49,999$, or $\$ 50,000+$. Physical Activity was measured using the Physical Activity for the Elderly (PASE) scale and categorized into low, moderate, or high [31]. Anthropometric measures-Trained field Interviewers directly measured the subject's height $(\mathrm{cm})$, weight $(\mathrm{kg})$, from which BMI was calculated $\left(\mathrm{kg} / \mathrm{m}^{2}\right)$, and waist circumference (cm). Waist circumference was further dichotomized into high waist circumference defined as $\geq 88 \mathrm{~cm}$ for women and $\geq 102 \mathrm{~cm}$ for men $[32,33]$. BMI was dichotomized using the cut-off point of $25 \mathrm{~kg} / \mathrm{m}^{2}$ that defines overweight in the current WHO classification.

Diet was measured with the Block Food Frequency Questionnaire (FFQ) administered in English or Spanish. The FFQ has been validated to obtain data on usual dietary intake over the past year [34]. We calculated an overall healthy eating score using the USDA and AHA guidelines for healthy eating $[35,36]$. The healthy eating score is composed of FFQ data on average daily intake of sodium (g), vegetables (servings/day), fruits (servings/day), meats/beans (serving/day), grains (servings/day), fiber (g), and saturated fat (g). Participants were classified into three possible diet score groupings: poor (score 0-2 points), intermediate (score 2-5 points), or high (score 5-7 points). The overall healthy eating index for this sample ranged from 0 to 5 indicating that no one met criteria for high (healthiest) healthy eating score. Therefore, for analyses, we created 3 groups (low/medium/high) based on off tertile cut-points. Each individual component of the diet score was also examined separately.

Sleep duration was measured using self-reported sleep patterns in the previous month with the question "How many hours of actual sleep do you get during the night?" and grouped as follows: $<6.5$ hours, $6.5-<8$ hours, and $\geq 8$ hours to create low, intermediate, and high duration of sleep.

Multiple risk factors measures were created by combining diet, sleep, and activity scores. The presence of two factors is defined as having poor diet (lowest tertile) and low sleep duration ( $<6.5$ hours). The three-risk-factor measure is defined as the presence of low physical activity, poor diet, and low sleep duration. Lastly the four-risk-factor measure is defined as the presence of high BMI $(\geq 25)$ or high waist circumference ( $\geq 88 \mathrm{~cm}$ women and $\geq 102 \mathrm{~cm}$ men) and presence of the three risk factors mentioned above. 
2.3. Major Outcome Measures. Diagnosed type 2 diabetes was determined by self-report (Have you ever been told by a doctor or other health professional that you have type 2 diabetes?). Medication inventory confirmed over $80 \%$ of the self-reported cases of diabetes. Insulin resistance was measured using the homeostasis model assessment (HOMA) index where the product of fasting glucose and fasting insulin was taken and then divided by 405 [37]. Insulin resistance was calculated only in subjects that had fasting glucose collected and has an analytic sample size of 2359. Prediabetes is defined among those subjects with no diabetes (type 1 or type 2 ) and no undiagnosed diabetes defined as fasting glucose $>125$ or $\mathrm{HbAlc} \geq 6.5$. Subjects were considered prediabetic if they have fasting glucose 100-125 or HbAlc 5.7-6.4 [38]. Subjects with prediabetes are compared to diabetes unaffected subjectsthat is, those without diabetes (self-reported type 1 or 2 or undiagnosed) and with no prediabetes leaving an analytic sample size of 2175. A third outcome combining prediabetes and insulin resistance was created to capture the presence of prediabetes or insulin resistance among nondiabetic subjects $(N=2175)$. To define insulin resistance, we used the NHANES study cut-point of $\geq 2.73$ [39].

2.4. Statistical Analysis. Bivariate associations between health factors and education were examined with Wald $F$ Chi-Square tests for categorical measures and Wald F test $P$ value from linear regression models for continuous measures.

Logistic regression models were utilized to examine the association of lifestyle/behavioral health factors with prediabetes and the combined outcome of insulin resistance or prediabetes. Linear regression models were constructed to examine the association between lifestyle and behavioral factors on insulin resistance. Due to positive skew, insulin resistance was log transformed in final regressions. In all regression models we stratified by education level or income group and adjusted for age, gender, race/ethnicity, family history of diabetes, and smoking status. Regression models were examined for the overall healthy eating score as well as each of the 7 subscales but results are presented only for the overall score. Analyses of diet were adjusted for total caloric intake to minimize measurement error by over/underreporting [40]. Separate unstratified regression models were examined to test for interactions between lifestyle and behavioral factors and SES measures income and education.

All analyses were performed with SAS callable SUDAAN 11.0 [41] using sampling weights and stratification measures to account the complex survey design of $\mathrm{BACH}$. Multiple imputation was used to reduce bias resulting from missing data for all exposure covariates using multivariate imputation by chained equations (MICE) in R [3]. Briefly, MICE imputes missing values with estimated predictions from regression models that reflect the relationships observed in the data, while considering the complex survey sampling design. Age, race, and their interaction were included as predictors in each of the imputation models. In addition, mice selected important predictors for each variable and these were also included in the model. Less than $5 \%$ of the covariates (age, gender, education, race/ethnicity, and income) were imputed [42]. Fifteen multiple imputation datasets were created stratified by race/ethnicity by gender.

\section{Results and Discussion}

The study population characteristics are shown in Table 1. Among the total sample, $28 \%$ had normal blood glucose levels (without self-reported diabetes and not prediabetic). In the sample without diabetes $(n=2379) 65 \%$ were prediabetic and $69 \%$ were prediabetic or IR. The geometric mean of the HOMA-IR measure was 1.9 (SE 1.0). Among those with prediabetes, over half were White and reported medium levels of physical activity and $46 \%$ reported a family history of diabetes. In subjects with prediabetes or IR, $45 \%$ were male and reported a family history of diabetes. The mean waist circumference for those with prediabetes or IR is the highest at $96 \mathrm{~cm}$ compared to those unaffected or with prediabetes alone. In general, mean diet FFQ scores (total score and subscales) were comparable across groups.

Similar patterns were observed in the bivariate associations between study population characteristics with educational level and income (data not shown). Educational level is significantly associated with most measures except for total FFQ score. Males were more likely to have higher education (postgraduate) compared to female (51\% versus $49 \%, P=0.04)$. The $<$ HS group also reported the highest rates of current smoking and family history of diabetes compared to all other education levels $(P \leq 0.0001$ in all comparisons). Regarding diabetes outcomes, subjects in the highest education group had higher rates of being in the unaffected group (34\% versus $12 \%, P \leq 0.0001$ ) and lower rates of reporting prediabetes $(61 \%$ versus $80 \%, P \leq 0.0001)$ and prediabetes or IR ( $65 \%$ versus $83 \%, P=0.006)$ compared to the lowest education group. Gender, current smoking, family history and diabetes, and the diabetes outcomes were associated with income with similar patterns.

In unstratified models there were no significant interactions seen between education and income with lifestyle/behavioral factors (data not shown). Table 2 displays the results of the insulin resistance regressions and Figure 1 plots mean HOMA-IR for significant predictors. Insulin resistance is associated with BMI and waist circumference across all levels of education and income ( $P$ ranges from 0.02 to $\leq 0.0001$, Table 2, Models 2 and 3). The magnitudes of association with BMI and waist circumference on IR are similar across all levels of education and income. Those with $\mathrm{BMI} \geq 25$ have on average a higher mean log IR score of 0.20 compared to those with BMI $<25$. Similarly, those with a high waist circumference have higher mean IR scores anywhere between 0.22 and 0.33 points higher than those with a smaller waist circumference. The healthy eating score, other diet components, physical activity, and sleep duration were not associated with insulin resistance. In addition, presence of multiple risk factors was not predictive of insulin resistance.

The covariate adjusted geometric means for insulin resistance in the $\mathrm{BMI} \geq 25$ group are nearly twice as high 
TABLE 1: Characteristics of BACH-3 analytic sample $(N=3155)$, overall and by fasting glucose/diabetes status.

\begin{tabular}{|c|c|c|c|c|c|}
\hline & & \multicolumn{4}{|c|}{ Fasting glucose/diabetes status } \\
\hline & $\begin{array}{l}\text { Total } \\
N=3155\end{array}$ & $\begin{array}{c}\text { Unaffected } \\
N=641(27.7 \%)\end{array}$ & $\begin{array}{c}\text { Prediabetes }^{* * *} \\
N=1533(64.6 \%)\end{array}$ & $\begin{array}{c}\text { Prediabetes or } \\
\text { insulin resistant } \\
N=1627(69.2 \%)^{* * *}\end{array}$ & $\begin{array}{c}\text { Insulin resistance } \\
\text { HOMA model }^{*} \\
\quad N=2359 \\
\text { Mean }(\mathrm{SE})=1.9(1.0)\end{array}$ \\
\hline Age, y mean SE & $54.0(0.5)$ & $50.8(0.8)$ & $53.7(0.6)$ & $53.5(0.6)$ & 0.05 \\
\hline \multicolumn{6}{|l|}{ Gender, \% } \\
\hline Male & $1184(46.5 \%)$ & $241(47.8 \%)$ & $559(43.9 \%)$ & $599(44.5 \%)$ & $2.04(1.05)$ \\
\hline Female & $1971(53.5 \%)$ & $399(52.2 \%)$ & $973(56.1 \%)$ & $1028(55.5 \%)$ & $1.73(1.05)$ \\
\hline BMI, mean SE & $29.5(0.2)$ & $27.6(0.3)$ & $29.3(0.3)$ & $29.4(0.3 \%)$ & 0.41 \\
\hline Waist circumference, $\mathrm{cm}$ mean SE & $97.0(0.5)$ & $91.9(1.0)$ & $95.8(0.7)$ & $96.2(0.6 \%)$ & 0.45 \\
\hline \multicolumn{6}{|l|}{ Education } \\
\hline$<\mathrm{HS}$ & $618(7.9 \%)$ & $76(3.4 \%)$ & $246(7.2 \%)$ & $259(7.0 \%)$ & $2.24(1.09)$ \\
\hline HS or GED & $948(24.4 \%)$ & $173(21 \%)$ & $454(23.1 \%)$ & $483(23.1 \%)$ & $2.24(1.06)$ \\
\hline Some college & $671(20.5 \%)$ & $139(18.2 \%)$ & $332(21 \%)$ & $354(21.2 \%)$ & $2.06(1.07)$ \\
\hline College or advanced & $916(47.1 \%)$ & $251(57.4 \%)$ & $499(48.7 \%)$ & $531(48.8 \%)$ & $1.60(1.05)$ \\
\hline \multicolumn{6}{|l|}{ Income } \\
\hline$<\$ 20,000$ & $1338(27.0 \%)$ & $204(19.1 \%)$ & $577(23.8 \%)$ & $615(24.5 \%)$ & $2.43(1.07)$ \\
\hline$\$ 20,000-\$ 49,999$ & $914(25.1 \%)$ & $181(21.2 \%)$ & $472(27.1 \%)$ & $498(26.7 \%)$ & $1.97(1.05)$ \\
\hline$>\$ 50,000$ & $902(48.0 \%)$ & $93(59.7 \%)$ & $482(49.2 \%)$ & $514(48.8 \%)$ & $1.68(1.07)$ \\
\hline \multicolumn{6}{|l|}{ Race/ethnicity } \\
\hline Black & $1026(27.1 \%)$ & $140(13.8 \%)$ & $504(29.1 \%)$ & $531(28.6 \%)$ & $2.30(1.06)$ \\
\hline Hispanic & $1036(12.2 \%)$ & $195(11.6 \%)$ & $475(12.5 \%)$ & $507(12.7 \%)$ & $1.96(1.08)$ \\
\hline White & $1093(60.7 \%)$ & $305(74.6 \%)$ & $553(58.4 \%)$ & $589(58.7 \%)$ & $1.69(1.05)$ \\
\hline \multicolumn{6}{|l|}{ Physical activity } \\
\hline Low & $1244(32.2 \%)$ & $185(25 \%)$ & $546(30.7 \%)$ & $580(30.8 \%)$ & $2.14(1.06)$ \\
\hline Medium & $1492(51.2 \%)$ & $346(57 \%)$ & $768(52.3 \%)$ & $812(51.9 \%)$ & $1.75(1.05)$ \\
\hline High & $417(16.6 \%)$ & $109(18 \%)$ & $218(17 \%)$ & $235(17.3 \%)$ & $1.75(1.08)$ \\
\hline \multicolumn{6}{|l|}{ Smoking status } \\
\hline Never & $1386(45.2 \%)$ & $295(48.5 \%)$ & $685(46.3 \%)$ & $727(46.3 \%)$ & $1.83(1.05)$ \\
\hline Former & $1160(36.2 \%)$ & $231(35.6 \%)$ & $541(35 \%)$ & $573(34.8 \%)$ & $1.85(1.05)$ \\
\hline Current & $608(18.6 \%)$ & $113(15.9 \%)$ & $306(18.7 \%)$ & $327(18.9 \%)$ & $2.03(1.09)$ \\
\hline Total Diet Quality score** & $0.67[0.02,1.42]$ & $0.68[0.03,1.43]$ & $0.69[0.03,1.43]$ & $0.69[0.04,1.43]$ & -0.02 \\
\hline \multicolumn{6}{|l|}{ Sleep duration } \\
\hline$<6.5 \mathrm{HR}$ & 1477 (41.9\%) & $238(32.7 \%)$ & $706(41.4 \%)$ & $740(40.6 \%)$ & $2.08(1.05)$ \\
\hline $6.5-<8.0 \mathrm{HR}$ & $881(32.4 \%)$ & $221(41 \%)$ & $443(31.5 \%)$ & $473(31.7 \%)$ & $1.61(1.06)$ \\
\hline$\geq 8.0 \mathrm{HR}$ & $795(25.7 \%)$ & $181(26.3 \%)$ & $383(27.2 \%)$ & $414(27.7 \%)$ & $1.89(1.06)$ \\
\hline HOMA-IR* & $1.86(1.05)$ & $1.27(1.05)$ & $1.86(1.04)$ & $2.68(0.12)$ & NA \\
\hline Family history of diabetes & $1686(46.5 \%)$ & $276(36.8 \%)$ & $751(45.7 \%)$ & $792(45.2)$ & $2.09(1.05)$ \\
\hline
\end{tabular}

${ }^{*}$ Geometric means are presented for categorical variables and weighted Pearson's correlations are presented for continuous variables. Insulin resistance was collected on subjects with fasting glucose.

${ }^{* *}$ Median [interquartile range] presented for skewed predictors.

${ }^{* * *}$ Conducted among $N=2175$ nondiabetic subjects.

compared to the BMI $<25$ group within the lowest education level $<$ HS or GED (Figure 1). The same magnitude in mean differences between the BMI categories is also seen in the HS or GED and some college group. Geometric HOMA-IR means are also higher in those with $\mathrm{BMI} \geq 25$ compared to $\mathrm{BMI}<25$ within income levels. Across both education and income groups, HOMA-IR geometric means are lower as one moves into higher educated or income groups. The geometric means for those with $\mathrm{BMI} \geq 25$ are $2.8,2.2$, and 1.8 for the income groups $<\$ 20,000, \$ 20,000-\$ 49,000$, and $\$ 50,000+$, respectively. With regard to waist circumference, higher mean HOMA-IR values are observed among subjects 


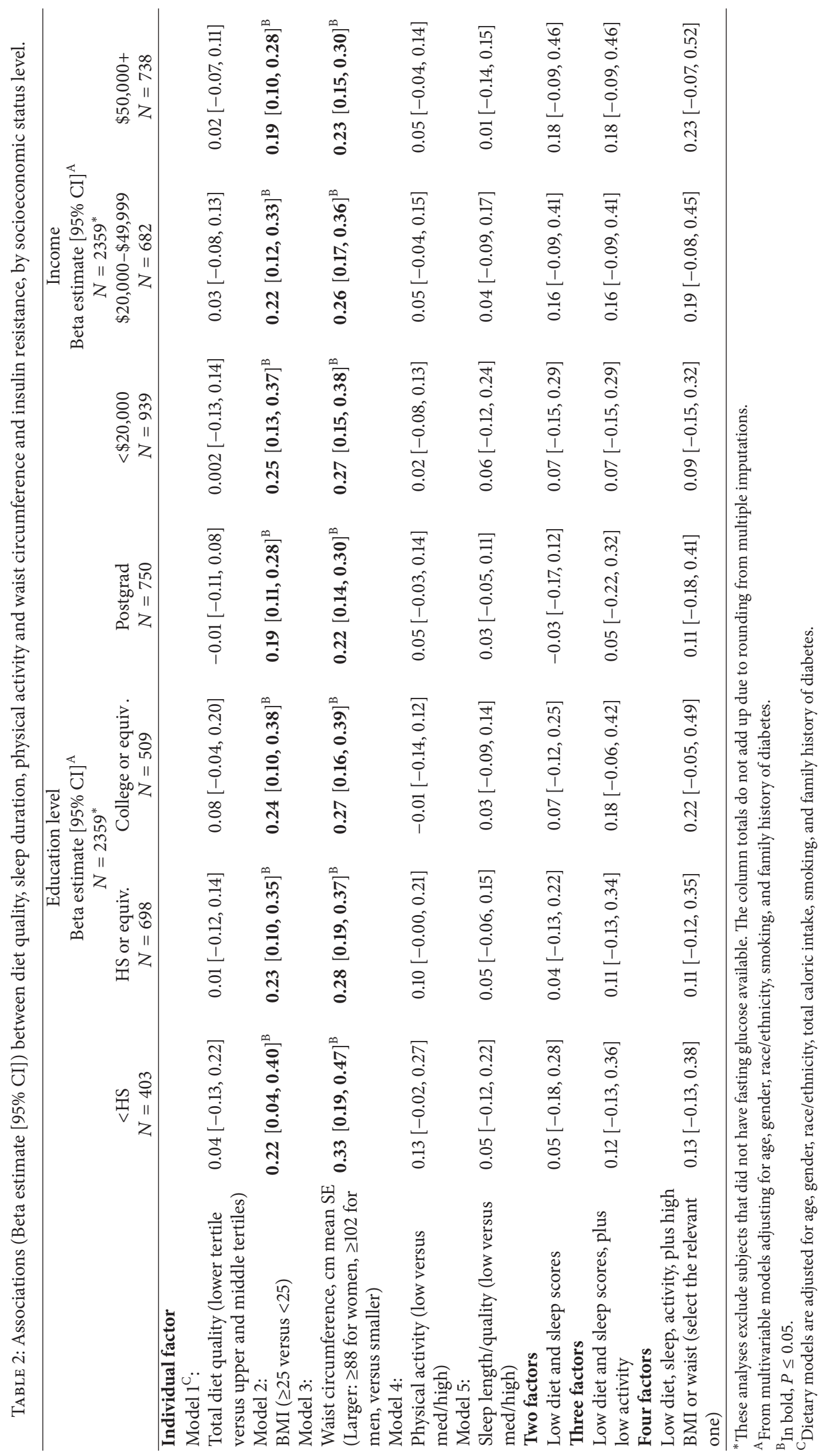




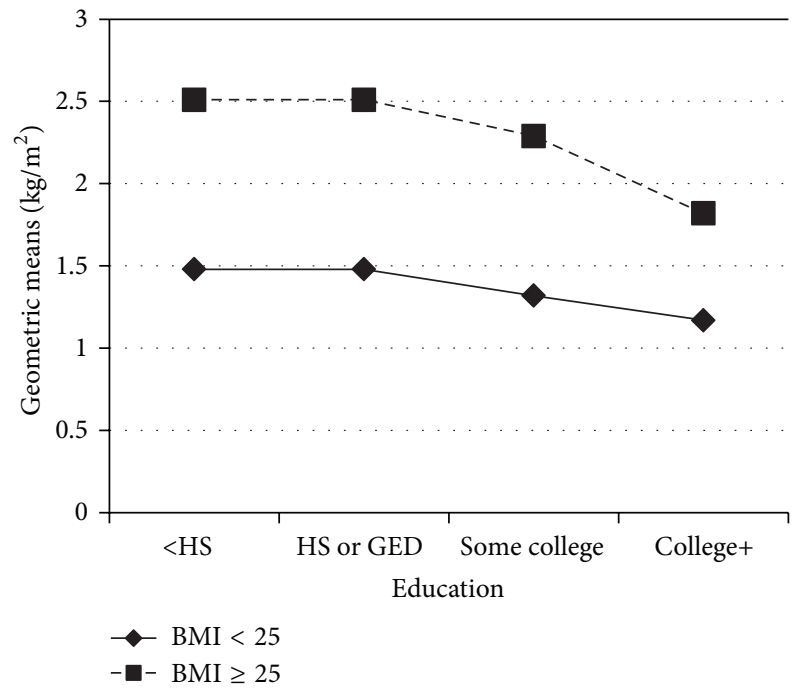

(a)

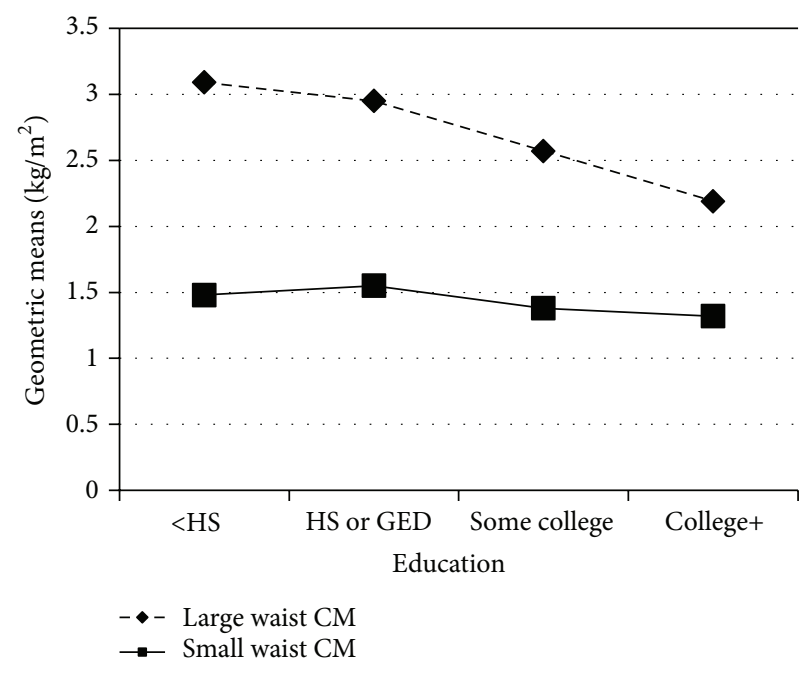

(c)

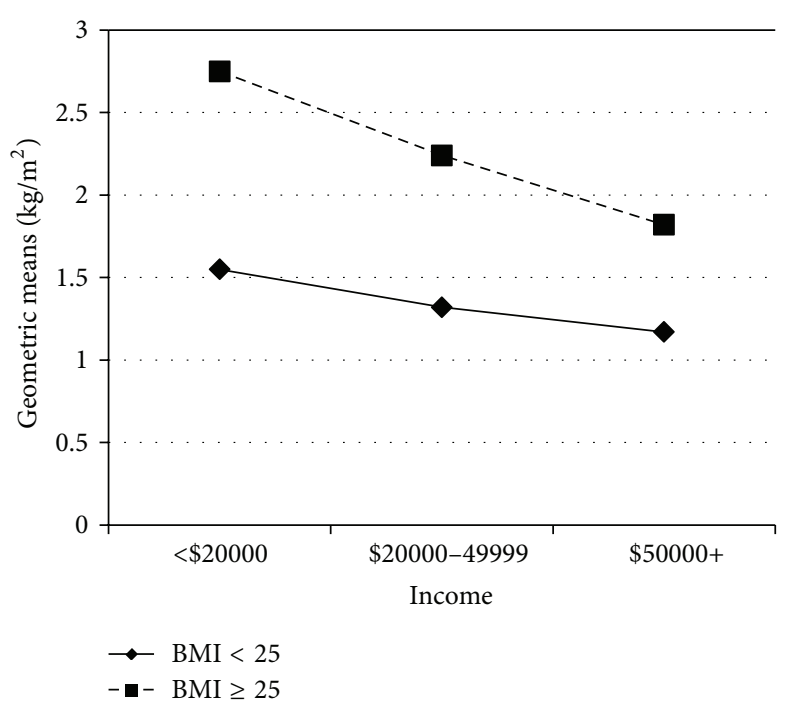

(b)

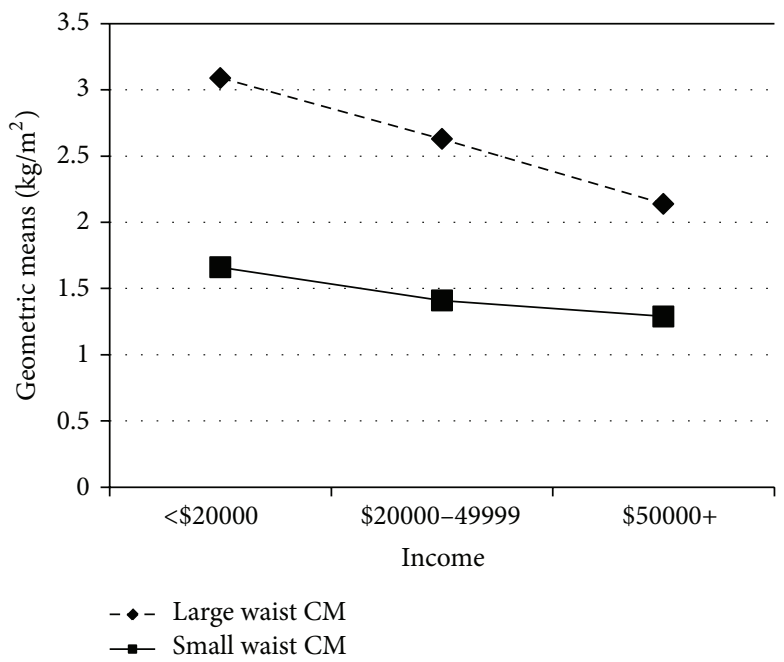

(d)

FIGURE 1: Geometric means of BMI and waist circumference by education and income strata.

with large waist $\mathrm{cm}$ compared to small waist $\mathrm{cm}$ within all levels of education and income ( $P \leq 0.0001$ in all models). These differences are nearly threefold.

Few significant associations were seen in logistic regression models of prediabetes (Table 3). Large waist circumference was significantly associated with increased odds of prediabetes in the college or advance degree group such that those with large waist circumference were 1.7 times as likely to develop prediabetes compared to those with lower waist circumference (OR 1.68, 95\% CI: 1.07-2.62). Similarly for income, subjects with large waist $\mathrm{cm}$ were nearly twice as likely to have prediabetes compared to those of lower waist $\mathrm{cm}$ in the medium and high income strata with OR 2.13 (95\% CI: 1.10-4.10), and OR 1.88 (95\% CI: 1.22-2.92) for medium and high income respectively. As with insulin resistance, diet, physical activity, sleep duration, and the presence of multiple risk factors were not associated with prediabetes.
Figures 2 and 3 display the results of the prediabetes or IR logistic regression models for strata of education and income, respectively. We see similar patterns to the insulin resistance and prediabetes models. Waist circumference remained a significant predictor of prediabetes or IR. Subjects with a large waist circumference were nearly twice as likely to develop prediabetes or IR in the higher education strata (some college and college+) and among the highest income group $(\$ 50,000+)$. The association with multiple risk factors remained nonsignificant. The odds ratios for large waist circumference are in the same positive direction and of similar magnitude across all education levels with a range of 1.55 to 1.88 , where a larger waist circumference nearly doubles the likelihood of prediabetes or IR. However the association in the <HS and HS or GED strata is nonsignificant. The same strength of association with waist circumference is seen between income strata, where the odds ratios range between 


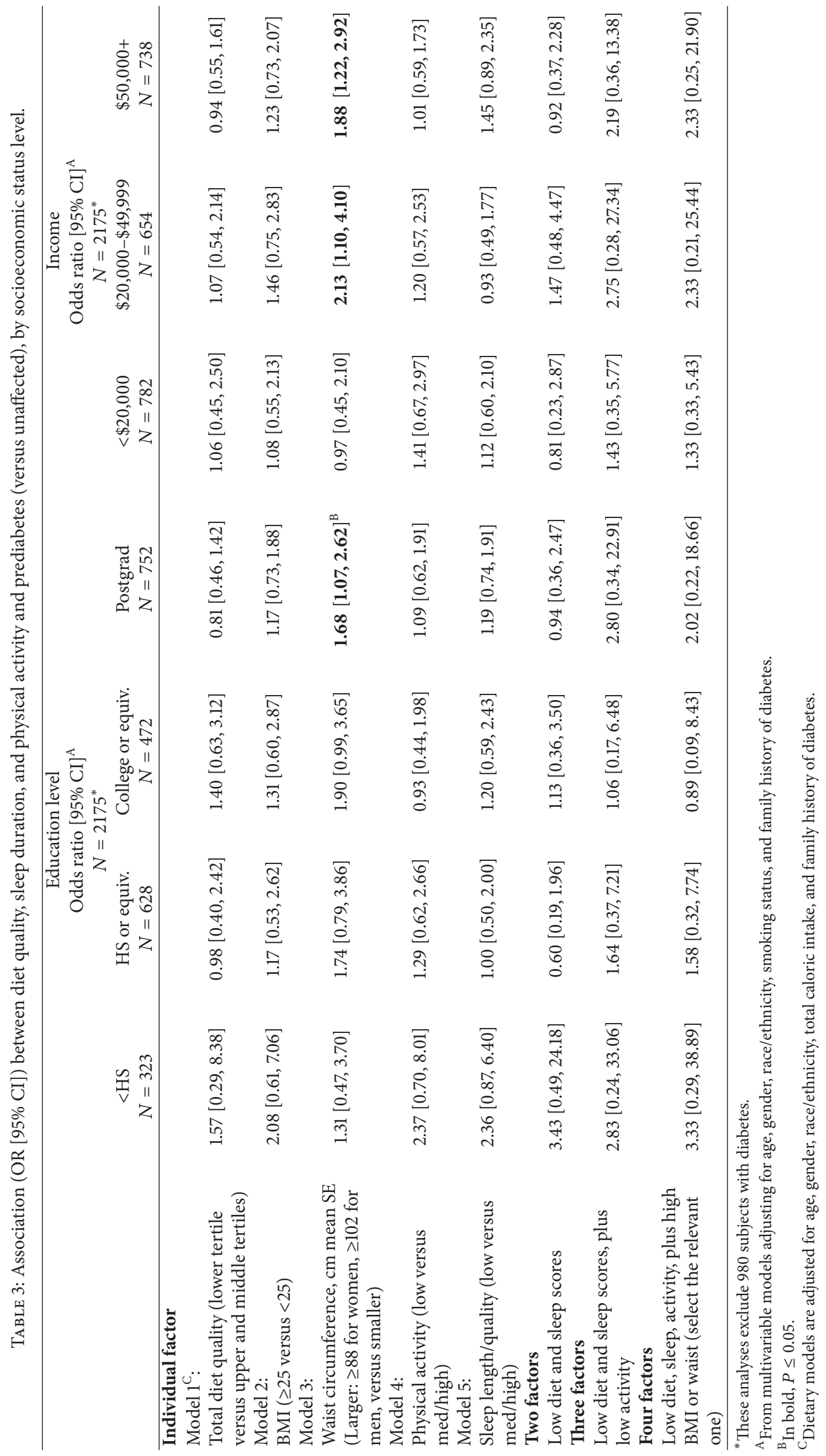




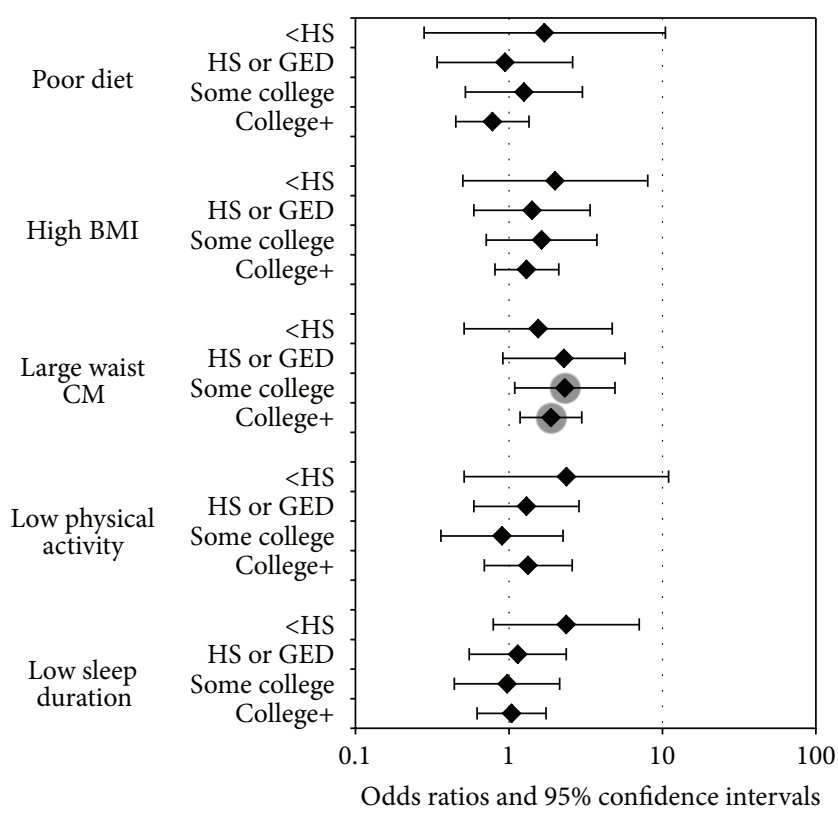

FIGURE 2: Odds ratio estimates for prediabetes or insulin resistance by education strata.

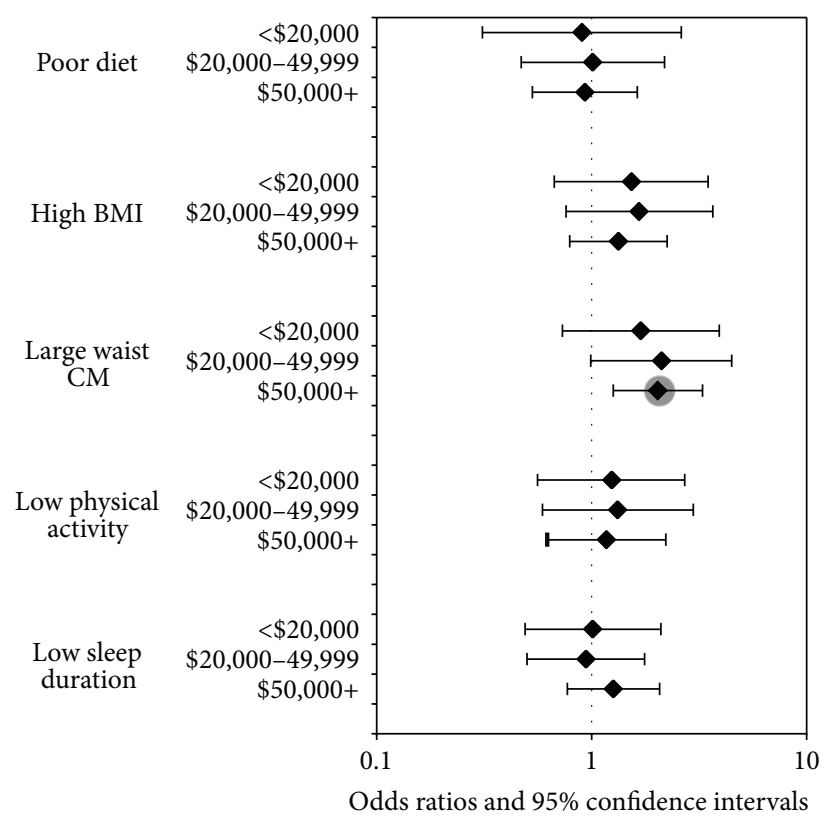

FIgURE 3: Odds ratio estimates for prediabetes or insulin resistance by income strata.

1.69 and 2.11 and reached significance only within the highest income strata.

\section{Conclusions}

Our analysis shows that, after adjusting for SES indicators (education and income), commonly known diabetes behavioral risk factors (namely, diet quality, sleep duration, and physical activity) are poor predictors of prediabetes and insulin resistance. Larger waist circumference was a consistent predictor of prediabetes and insulin resistance with large waist circumference increasing the probability of prediabetes or IR nearly twofold. While this magnitude of effect is seen across all levels of SES, it reaches significance primarily within higher SES levels. The examination of multiple risk factors revealed that the presence of two or more risk factors is not associated with prediabetes or IR. While many studies have shown significant associations between diet and diabetes risk $[17,27,43-45]$, these results have been mixed. For example, other studies have shown no association between diet quality, specifically total fat intake and red meat are not predictive of diabetes risk $[46,47]$. Other studies have shown that BMI 
adjustment attenuates the association between diet quality and type 2 diabetes [48-50] which supports our findings of BMI being a significant predictor of insulin resistance across all levels of SES.

The significant findings between BMI and waist circumference indicate that, independent of education and income, increased BMI and waist circumference are important predictors of prediabetes and insulin resistance. The strength of our waist circumference results is corroborated by a study which examined the interrelationships between demographic (age, income, marital, race, and education) and physical activity and poor diet on prediabetes in path models, concluding that large waist circumference had the strongest direct effect on prediabetes [51]. In addition, findings from the NHANES 2009-2010 survey indicated that the significance of sleep disorders on diabetes is attenuated when BMI is added to the model, where the odds ratio for diabetes drops from 2.04 $(1.40,2.95)$ to $1.38(0.95,2.00)$. They conclude that the effect of sleep disorders on diabetes may be explained through a subject's obesity status [52]. While they controlled for various factors such as age, gender, ethnicity, education, and income, they did not examine other modifiable risk factors (e.g., diet and sleep).

The significant association of waist circumference with both prediabetes and insulin resistance may help guide future primary and secondary prevention programs where, in addition to socioeconomic factors, subjects with high BMI and large waist circumference are likely to produce the most beneficial outcomes. The finding that waist circumference is not predictive across all education levels in prediabetes suggests that there may be different predictors of prediabetes among the lower SES groups providing new opportunities for more targeted intervention programs. On a broader level, our analyses showed no association between known diabetes risk factors and prediabetes. This may be explained by the fact that prediabetes is considered an early precursor state indicating likely eventual development of diabetes, and not the eventual established diabetes state itself, by which time the associated risk factors are more evident and strongly associated. Moreover, while the majority of prediabetic cases may end up being diagnosed with diabetes, not all cases will be; therefore the expected relationship of known diabetes risk factors with prediabetes is diluted.

There are some limitations to this epidemiologic investigation. First, because it is cross-sectional; the temporality of the relationships uncovered remains uncertain. Fasting insulin was also only measured at a single time point and maybe subject to measurement error. The fact that measures are obtained at a single time point may reduce the likelihood of finding significant differences as research has shown that repeated measures of health behaviors may increase the significance and the effect size of such modifiable behaviors [53]. Physical, direct measures such as BMI and waist circumference may serve as a lifetime proxy for diet quality and level of physical activity compared to self-reported measures of health behaviors, taken at a single time point. While the reliability of self-reports of health conditions may be questioned, there is evidence that they are generally wellcorrelated with medical record review [54-57]. Fortunately, medication data were available and over $80 \%$ of those selfreporting diabetes were taking a diabetes-related medication.

Countervailing strengths of this study include use of a community-based random sample and the composition of the sample, which covers a broad age range, inclusion of both genders, and a racial/ethnic diversity, with roughly equal numbers of Blacks, Whites, and Hispanic participants. Inclusion of a broad range of recognized behavioral risk factors permitted assessment of their independent and joint influences on prediabetic states. Finally, we examined a variety and combination of multiple risk factors, sleep duration, diet, and physical activity to test whether targeting multiple behaviors were predictive of prediabetes or IR. We found that, among the behavioral risk factors considered, BMI and waist circumference were consistent predictors of prediabetes outcomes independent of SES. Our results have both clinical and public health significance: many different risk factors (including BMI and waist circumference) are variably associated with diabetes, prediabetes, and IR and offer variably effective opportunities for primary and secondary prevention. By identifying, among the broad range of risk factors, the most promising influences, future primary and secondary prevention initiatives can be more precisely targeted, resulting in more effective and cost efficient outcomes.

\section{Conflict of Interests}

The authors declare that there is no conflict of interests regarding the publication of this paper.

\section{Acknowledgments}

This paper was supported by a grant from the National Institutes of Health (DK080786). The National Institute of Diabetes and Digestive and Kidney Diseases had no role in the design and conduct of the study, in the collection, analysis, and interpretation of the data, or in the preparation, review, or approval of the paper.

\section{References}

[1] Centers for Disease Control and Prevention, Ed., National Diabetes Fact Sheet: National Estimates and General Information on Diabetes and Prediabetes in the United States, Centers for Disease Control and Prevention, Atlanta, Ga, USA, 2001.

[2] J. E. Shaw, R. A. Sicree, and P. Z. Zimmet, "Global estimates of the prevalence of diabetes for 2010 and 2030," Diabetes Research and Clinical Practice, vol. 87, no. 1, pp. 4-14, 2010.

[3] "Awareness of prediabetes-United States, 2005-2010," Morbidity and Mortality Weekly Report, vol. 62, no. 11, pp. 209-212, 2013.

[4] J. P. Boyle, T. J. Thompson, E. W. Gregg, L. E. Barker, and D. F. Williamson, "Projection of the year 2050 burden of diabetes in the US adult population: dynamic modeling of incidence, mortality, and prediabetes prevalence," Population Health Metrics, vol. 8, article 29, 2010.

[5] G. N. Ioannou, C. L. Bryson, and E. J. Boyko, "Prevalence and trends of insulin resistance, impaired fasting glucose, and diabetes," Journal of Diabetes and its Complications, vol. 21, no. 6, pp. 363-370, 2007. 
[6] A. J. Garber, Y. Handelsman, D. Einhorn et al., "Diagnosis and management of prediabetes in the continuum of hyperglycemia - when do the risks of diabetes begin? A consensus statement from the American College of Endocrinology and the American Association of Clinical Endocrinologists," Endocrine Practice, vol. 14, no. 7, pp. 933-946, 2008.

[7] D. M. Nathan, M. B. Davidson, R. A. DeFronzo et al., "Impaired fasting glucose and impaired glucose tolerance: implications for care," Diabetes Care, vol. 30, no. 3, pp. 753-759, 2007.

[8] L. S. Geiss, C. James, E. W. Gregg, A. Albright, D. F. Williamson, and C. C. Cowie, "Diabetes risk reduction behaviors among U.S. adults with prediabetes," The American Journal of Preventive Medicine, vol. 38, no. 4, pp. 403-409, 2010.

[9] "Symptoms," http://www.diabetes.org/diabetes-basics/symptoms/.

[10] National Cholesterol Education Program Expert Panel on Detection Evaluation-Treatment of High Blood Cholesterol in Adults, "Third report of the National Cholesterol Education Program (NCEP) Expert Panel on Detection, Evaluation, and Treatment of High Blood Cholesterol in Adults (Adult Treatment Panel III) final report," Circulation, vol. 106, pp. 3143-3421, 2002.

[11] R. A. Miech and R. M. Hauser, "Socioeconomic status and health at midlife: a comparison of educational attainment with occupation-based Indicators," Annals of Epidemiology, vol. 11, no. 2, pp. 75-84, 2001.

[12] J. Morgan and C. Lininger, "Education and income: comment," The Quarterly Journal of Economics, vol. 78, no. 2, pp. 346-347, 1964.

[13] Centers for Disease Control and Prevention, Physical Activity and Health: Report of the Surgeon General, Centers for Disease Control and Prevention, Atlanta, Ga, USA, 1996.

[14] American Diabetes Association, J. P. Bantle, J. Wylie-Rosett et al., "Nutrition recommendations and interventions for diabetes: a position statement of the American Diabetes Association," Diabetes Care, vol. 31, pp. S61-S78, 2008.

[15] K. Kempf, W. Rathmann, and C. Herder, "Impaired glucose regulation and type 2 diabetes in children and adolescents," Diabetes/Metabolism Research and Reviews, vol. 24, no. 6, pp. 427-437, 2008.

[16] S. H. Ley, O. Hamdy, V. Mohan, and F. B. Hu, "Prevention and management of type 2 diabetes: dietary components and nutritional strategies," Lancet, vol. 383, no. 9933, pp. 1999-2007, 2014.

[17] K. Esposito and D. Giugliano, "Increased consumption of green leafy vegetables, but not fruit, vegetables or fruit and vegetables combined, is associated with reduced incidence of type 2 diabetes," Evidence-Based Medicine, vol. 16, no. 1, pp. 27-28, 2011.

[18] N. T. Ayas, D. P. White, W. K. Al-Delaimy et al., "A prospective study of self-reported sleep duration and incident diabetes in women," Diabetes Care, vol. 26, no. 2, pp. 380-384, 2003.

[19] F. P. Cappuccio, L. D’Elia, P. Strazzullo, and M. A. Miller, "Quantity and quality of sleep and incidence of type 2 diabetes: a systematic review and meta-analysis," Diabetes Care, vol. 33, no. 2, pp. 414-420, 2010.

[20] J. E. Gangwisch, S. B. Heymsfield, B. Boden-Albala et al., "Sleep duration as a risk factor for diabetes incidence in a large U.S. sample," Sleep, vol. 30, no. 12, pp. 1667-1673, 2007.

[21] H. K. Yaggi, A. B. Araujo, and J. B. McKinlay, "Sleep duration as a risk factor for the development of type 2 diabetes," Diabetes Care, vol. 29, no. 3, pp. 657-661, 2006.
[22] J. Engeda, B. Mezuk, S. Ratliff, and Y. Ning, "Association between duration and quality of sleep and the risk of prediabetes: evidence from NHANES," Diabetic Medicine, vol. 30, no. 6, pp. 676-680, 2013.

[23] C. L. Jackson, S. Redline, I. Kawachi, and F. B. Hu, "Association between sleep duration and diabetes in black and white adults," Diabetes Care, vol. 36, pp. 3557-3565, 2013.

[24] Y. Ma, J. R. Hebert, J. E. Manson et al., "Determinants of racial/ethnic disparities in incidence of diabetes in postmenopausal women in the U.S.: the women's health initiative 1993-2009," Diabetes Care, vol. 35, pp. 2226-2234, 2012.

[25] Y. Qiao, L. Tinker, B. C. Olendzki et al., "Racial/ethnic disparities in association between dietary quality and incident diabetes in postmenopausal women in the United States: the Women's Health Initiative 1993-2005," Ethnicity and Health, vol. 19, pp. 328-347, 2014.

[26] C. C. Cowie and M. S. Eberhardt, "Sociodemographic characteristics of persons with diabetes," in Diabetes in America, pp. 85-116, National Institutes of Health, Bethesda, Md, USA, 1995.

[27] A. F. Brown, A. G. Gross, P. R. Gutierrez, L. Jiang, M. F. Shapiro, and C. M. Mangione, "Income-related differences in the use of evidence-based therapies in older persons with diabetes mellitus in for-profit managed care," Journal of the American Geriatrics Society, vol. 51, pp. 665-670, 2003.

[28] A. J. Karter, A. Ferrara, J. A. Darbinian, L. M. Ackerson, and J. V. Selby, "Self-monitoring of blood glucose: language and financial barriers in a managed care population with diabetes," Diabetes Care, vol. 23, pp. 477-483, 2000.

[29] J. B. McKinlay and C. L. Link, "Measuring the urologic iceberg: design and implementation of the Boston Area Community Health (BACH) Survey," European Urology, vol. 52, pp. 389-396, 2007.

[30] R. S. Piccolo, A. B. Araujo, N. Pearce, and J. B. McKinlay, "Cohort profile: the Boston Area Community Health (BACH) survey," International Journal of Epidemioloy, vol. 43, pp. 42-51, 2014.

[31] R. A. Washburn, K. W. Smith, A. M. Jette, and C. A. Janney, “The Physical Activity Scale for the Elderly (PASE): development and evaluation," Journal of Clinical Epidemiology, vol. 46, pp. 153162, 1993.

[32] "According to waist circumference," in Guidelines on Overweight and Obesity: Electronic Textbook, NHLBI, 1998.

[33] K. G. M. M. Alberti, R. H. Eckel, S. M. Grundy et al., "Harmonizing the metabolic syndrome: a joint interim statement of the international diabetes federation task force on epidemiology and prevention; National heart, lung, and blood institute; American heart association; World heart federation; International atherosclerosis society; And international association for the study of obesity," Circulation, vol. 120, no. 16, pp. 1640-1645, 2009.

[34] G. Block, A. M. Hartman, and C. M. Dresser, "A data-based approach to diet questionnaire design and testing," The American Journal of Epidemiology, vol. 124, no. 3, pp. 453-469, 1986.

[35] Dietary Guidelines for Americans 2010, Government Printing Office, Washington, DC, USA, 2010.

[36] American Heart Association Nutrition Committee, A. H. Lichtenstein, L. J. Appel, and M. Brands, "Diet and lifestyle recommendations revision 2006: a scientific statement from the American Heart Association Nutrition Committee," Circulation, vol. 114, pp. 82-96, 2006.

[37] S. Javaheri, A. Storfer-Isser, C. L. Rosen, and S. Redline, "Association of short and long sleep durations with insulin 
sensitivity in adolescents," Journal of Pediatrics, vol. 158, no. 4, pp. 617-623, 2011.

[38] K. M. Bullard, S. H. Saydah, G. Imperatore et al., "Secular changes in U.S. prediabetes prevalence defined by hemoglobin A 1c and fasting plasma glucose: National Health and Nutrition Examination Surveys, 1999-2010," Diabetes Care, vol. 36, no. 8, pp. 2286-2293, 2013.

[39] A. E. Sumner and C. C. Cowie, "Ethnic differences in the ability of triglyceride levels to identify insulin resistance," Atherosclerosis, vol. 196, no. 2, pp. 696-703, 2008.

[40] W. C. Willett, L. Sampson, and M. J. Stampfer, "Reproducibility and validity of a semiquantitative food frequency questionnaire," The American Journal of Epidemiology, vol. 122, no. 1, pp. 51-65, 1985.

[41] SUDAAN User's Manual, Release 11.0 [Package Insert], Research Triangle Institute, Research Triangle Park, NC, USA, 2012.

[42] S. van Buuren and K. Groothuis-Oudshoorn, "Mice: multivariate imputation by chained equations in R," Journal of Statistical Software, vol. 45, no. 3, 2011.

[43] S. A. McNaughton, D. W. Dunstan, K. Ball, J. Shaw, and D. Crawford, "Dietary quality is associated with diabetes and cardio-metabolic risk factors," Journal of Nutrition, vol. 139, no. 4, pp. 734-742, 2009.

[44] P. Carter, L. J. Gray, J. Troughton, K. Khunti, and M. J. Davies, "Fruit and vegetable intake and incidence of type 2 diabetes mellitus: Systematic review and meta-analysis," British Medical Journal, vol. 341, Article ID c4229, 2010.

[45] A. M. Hodge, D. R. English, K. O’Dea, and G. G. Giles, “Dietary patterns and diabetes incidence in the Melbourne collaborative cohort study," The American Journal of Epidemiology, vol. 165, no. 6, pp. 603-610, 2007.

[46] J. Salmeron, F. B. Hu, J. E. Manson et al., "Dietary fat intake and risk of type 2 diabetes in women," American Journal of Clinical Nutrition, vol. 73, pp. 1019-1026, 2001.

[47] D. Aune, G. Ursin, and M. B. Veierød, "Meat consumption and the risk of type 2 diabetes: a systematic review and meta-analysis of cohort studies," Diabetologia, vol. 52, no. 11, pp. 2277-2287, 2009.

[48] R. M. van Dam, W. C. Willett, E. B. Rimm, M. J. Stampfer, and F. B. $\mathrm{Hu}$, "Dietary fat and meat intake in relation to risk of type 2 diabetes in men," Diabetes Care, vol. 25, no. 3, pp. 417-424, 2002.

[49] R. Villegas, X. O. Shu, Y.-T. Gao et al., "The association of meat intake and the risk of type 2 diabetes may be modified by body weight," International Journal of Medical Sciences, vol. 3, no. 4, pp. 152-159, 2006.

[50] A.-H. Harding, N. E. Day, K.-T. Khaw et al., "Dietary fat and the risk of clinical type 2 diabetes: the European prospective investigation of Cancer-Norfolk study," American Journal of Epidemiology, vol. 159, no. 1, pp. 73-82, 2004.

[51] B. H. Bardenheier, K. M. Bullard, C. J. Caspersen, Y. J. Cheng, E. W. Gregg, and L. S. Geiss, "Novel use of structural equation models to examine factors associated with prediabetes among adults aged 50 years and older: National Health and Nutrition Examination Survey 2001-2006," Diabetes Care, vol. 36, no. 9, pp. 2655-2662, 2013.

[52] J. Liu, J. Hay, and B. E. Faught, "The Association of Sleep Disorder, Obesity Status, and Diabetes Mellitus among US Adults-The NHANES, 2009-2010 survey results," International Journal of Endocrinology, vol. 2013, Article ID 234129, 6 pages, 2013.
[53] S. Stringhini, S. Sabia, M. Shipley et al., "Association of socioeconomic position with health behaviors and mortality," JAMA, vol. 303, pp. 1159-1166, 2010.

[54] M. M. Bergmann, T. Byers, D. S. Freedman, and A. Mokdad, "Validity of self-reported diagnoses leading to hospitalization: a comparison of self-reports with hospital records in a prospective study of American adults," American Journal of Epidemiology, vol. 147, pp. 969-977, 1998.

[55] Y. Okura, L. H. Urban, D. W. Mahoney, S. J. Jacobsen, and R. J. Rodeheffer, "Agreement between self-report questionnaires and medical record data was substantial for diabetes, hypertension, myocardial infarction and stroke but not for heart failure," Journal of Clinical Epidemiology, vol. 57, pp. 1096-1103, 2004.

[56] J. L. St Sauver, P. T. Hagen, S. S. Cha et al., "Agreement between patient reports of cardiovascular disease and patient medical records," Mayo Clinic Proceedings, vol. 80, pp. 203-210, 2005.

[57] L. M. Martin, M. Leff, N. Calonge, C. Garrett, and D. E. Nelson, "Validation of self-reported chronic conditions and health services in a managed care population," American Journal of Preventive Medicine, vol. 18, pp. 215-218, 2000. 


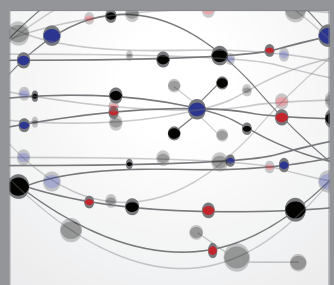

The Scientific World Journal
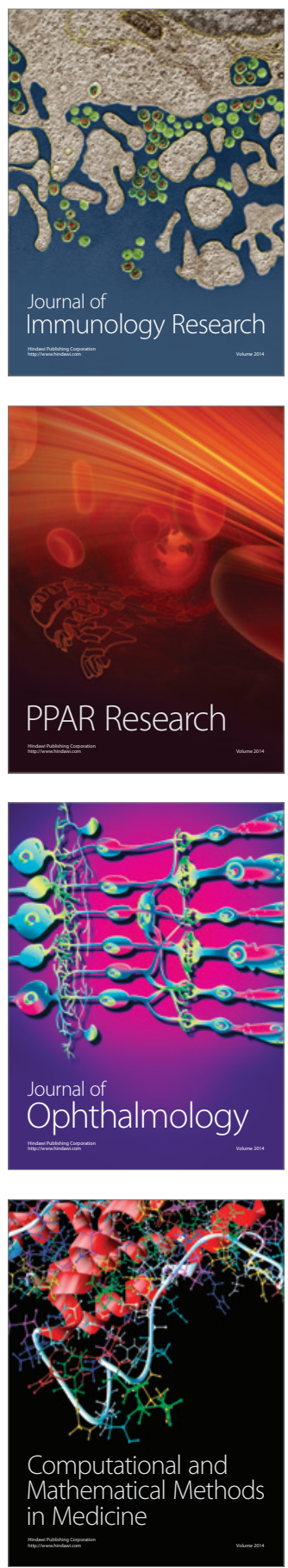

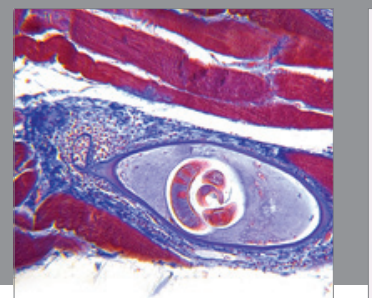

Gastroenterology

Research and Practice
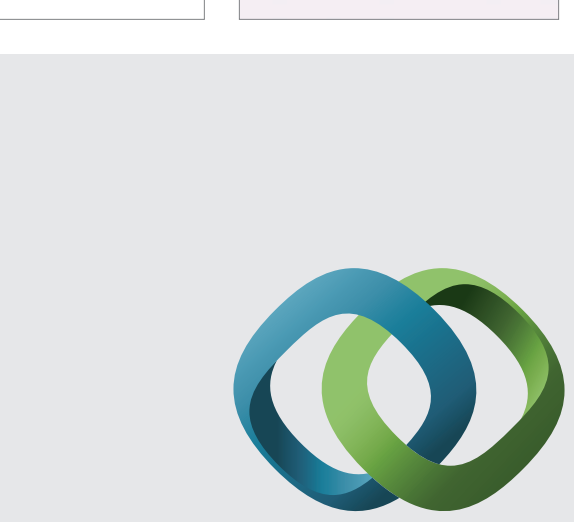

\section{Hindawi}

Submit your manuscripts at

http://www.hindawi.com
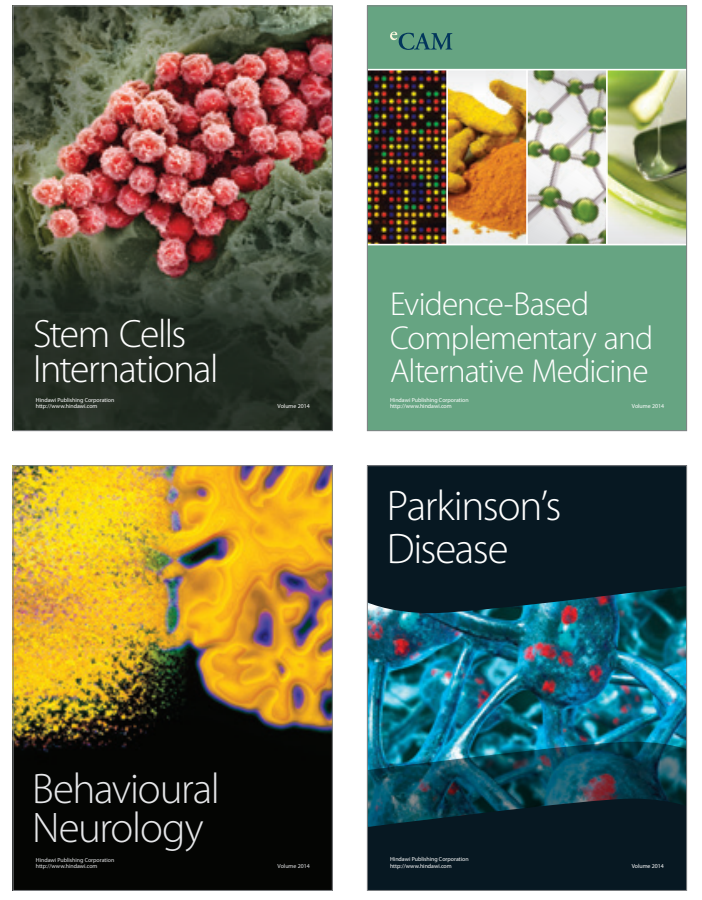
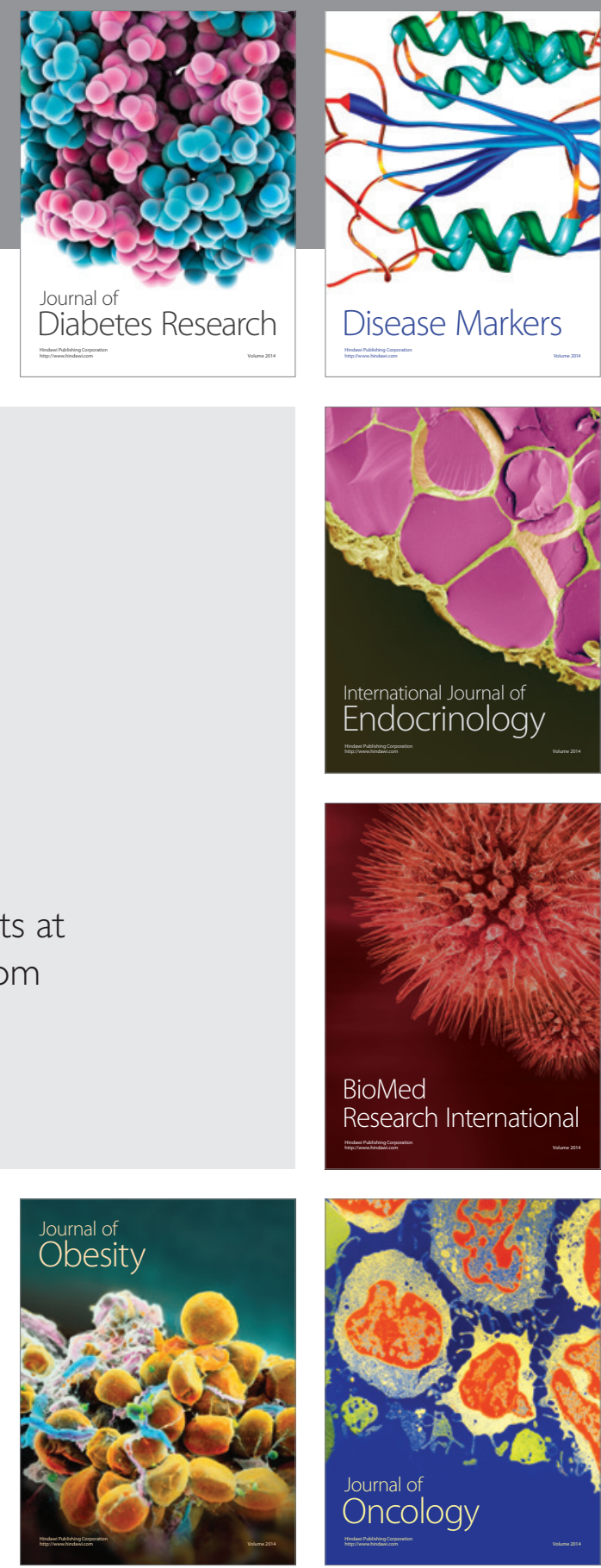

Disease Markers
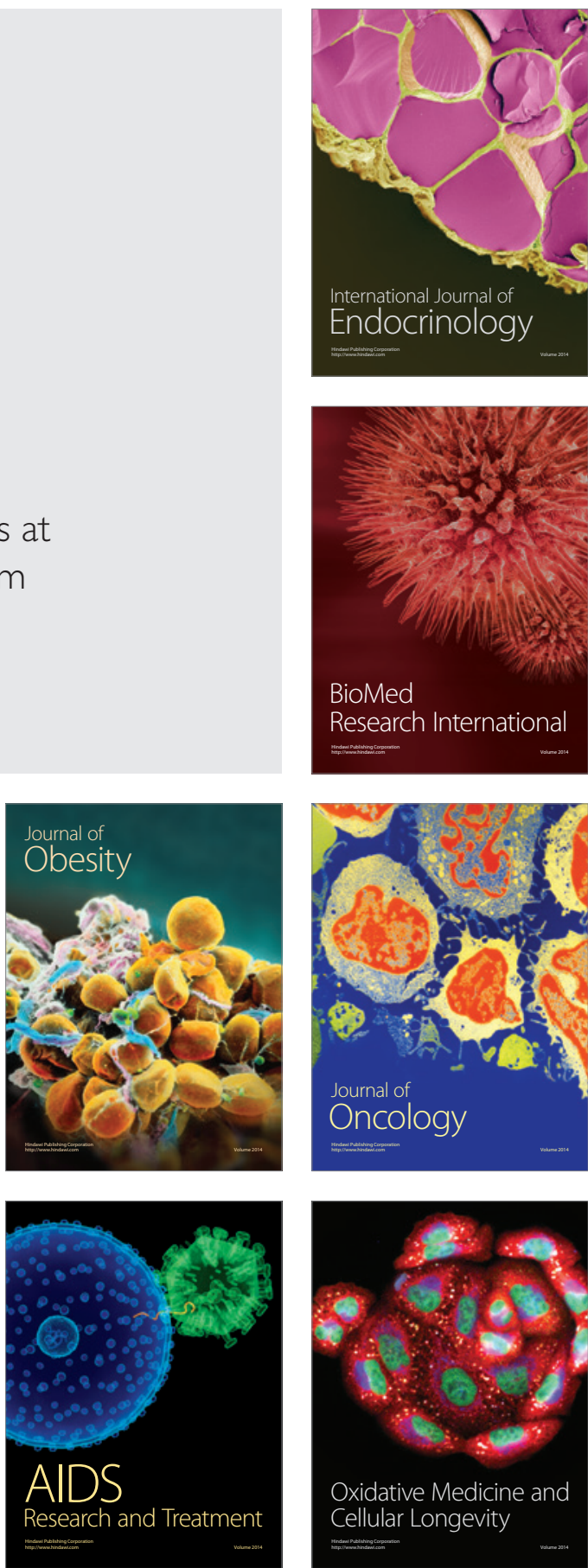